PharmacoEconomics \& Outcomes News 881, p8 - 26 Jun 2021

\title{
Costs of non-pharmaceutical interventions for infectious disease control
}

There are gaps in available data from previous studies on the direct costs of implementing public health nonpharmaceutical interventions (NPIs) for controlling infectious disease outbreaks, but findings from this review and cost studies on measures to control the COVID-19 pandemic may fill the gaps, say authors of a systematic review published in Applied Health Economics and Health Policy.

MEDLINE and EMBASE databases were searched for studies published between 1990 and March 2020 which reported cost data on NPIs in pandemic, epidemic, and outbreak response scenarios.

In total, 61 studies reporting relevant costs on NPIs of interest met the selection criteria; only $54 \%$ of these were assessed as being of high quality. Most of the studies were conducted in North America, Europe or Asia.

There was substantial heterogeneity in costs recorded for NPIs in outbreak cost studies. Direct costs of NPIs ranged from $\$ 141.18^{*}$ to $\$ 1042.68$ per case for isolating infected patients; from $\$ 40.73$ to $\$ 93.59$ per contact for tracing and quarantine of contacts; from $\$ 33.76$ to $\$ 167.92$ for social distancing; and from $\$ 0.15$ to $\$ 895.60$ for personal protection and hygiene.

"Having easily accessible collated cost information on community-based NPI strategies will provide a valuable resource for informing future outbreak response policies ... Additional care should also be taken to collect and publish costs for low-income settings for future planning of pandemic financing. Maintaining a database summarising published literature on NPI costs in relation to outbreak response could be valuable for model parameterisation and outbreak response planning purposes," said the authors.

* 2020 US dollars

Skarp JE, et al. A Systematic Review of the Costs Relating to Non-pharmaceutical Interventions Against Infectious Disease Outbreaks Applied Health Economics and Health Policy : 11 Jun 2021. Available from: URL: https://doi.org/10.1007/s40258-021-00659-Z 